\title{
Designing MEMS for manufacturing
}

\author{
Alexander Wolter*@ ${ }^{\circledR}$ Andreas Herrmann*, Gökhan Yildiz ${ }^{+}$, Harald Schenk*, Hubert Lakner* \\ *Fraunhofer of Institute Photonic Microsystems, \\ Grenzstr. 28, D-01099 Dresden, Germany \\ ${ }^{+}$KOC University Istanbul, Dept. of Electrical Engineering, \\ Rumeli Feneri Yolu, 80910 Sariyer, Turkey
}

\begin{abstract}
MEMS (micro electro-mechanical systems) are often expected to take a development as microelectronics did in the last 35 years. Several devices are already established in mass markets like acceleration sensors, gyros, pressure sensors, ink jet heads and the DLP micromirror array. On the other hand many companies have stopped their business after the telecom bubble. Others are struggling. Many dreams based on MEMS-devices that were not at all mature and could not be manufactured in high numbers. When a commercial product is the goal, several questions must be answered already in concept phase. The specifications must clearly reflect the requirements of the application. Performance and price must be competitive to any other technology.

The relation between fabrication process and design is strong and mutual. The process must create all features of the device and the design must consider the limitations of the process. Only if the design is tolerant against all process variations reproducible performance can be achieved. And only if the design is robust in all process steps the devices can survive. Regarding the time and cost frame it is always preferable to change the layout rather than the process. This article looks at MEMS technology and identifies what has been adopted from CMOS, what is desirable to adopt and what needs new solutions. Examples are given in the fields of design, modeling layout, process, test, and packaging.
\end{abstract}

Keywords: micro scanning mirror, micromirror array, optical MEMS, MOEMS, laser beam deflection, micromachining, out-of-plane comb drive, microsystem
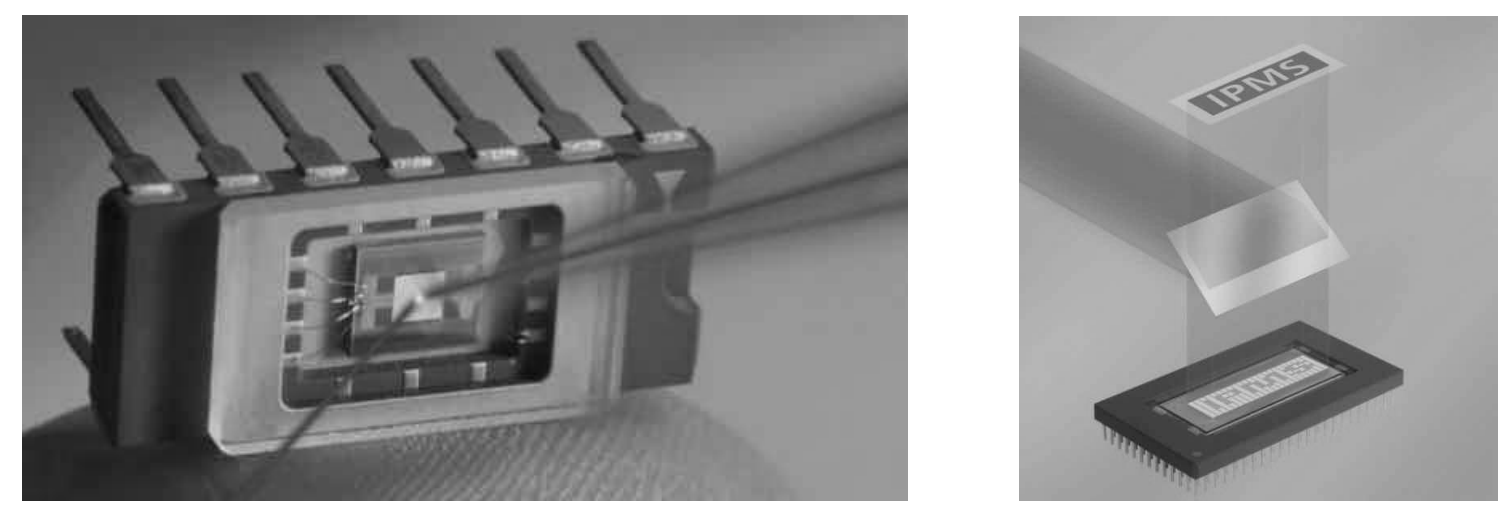

figure 1 Two examples for design for manufacture: The micro scanning mirror for laser beam deflection (left) and the micromirror array (right) for fast optical pattern generation with high resolution.

\footnotetext{
@ alexander.wolter@ipms.fraunhofer.de
} 


\section{INTRODUCTION}

System design is generally a complex subject. Functionality is the most obvious requirement, but there is more. The system must work under all operating conditions, e.g. temperature, humidity, vibration, or radiation. And it must work reliably under a certain time. The system must be fabricated in some way and certain numbers must be produced. It must be tested, transported, operated, maintained and finally disposed or recycled. System design has its impact on all of these stages. Good or bad design can reduce or increase efforts and subsequently cost significantly.

The design of microsystems ${ }^{*}$ and microelectronics is particularly complex because of the monolithic fabrication process. Monolithic fabrication allows only a limited number of degrees of freedom. The process must create all integrated features. Add-ons cannot be simply attached. On the other hand all features of the microsystem must pass the fabrication process. So the entire integrated system must work at one shot. Repair is hardly possible and only a very low number of redesigns can be considered, because every process run is expensive and turn-around times are counted in weeks or months. For this reason careful modeling is essential.

In CMOS the designer finds an established infrastructure. He does not need to overlook the entire complex field. He can consider the process as a black box described by design rules. Indeed the designer can chose from a number of available qualified processes that are completely developed and proven. He can "compose" his system on a high level of hierarchy from a toolbox with abstract functional standard blocks. Circuit design, modeling, layout generation, fabrication and test are a well-defined sequence.

The designer of microsystems faces two important differences: 1 . There is no such infrastructure for microsystems yet that provides versatile processes. The MEMS-law still says: "one device - one process" [1]. 2. It must be questioned if microsystems can ever be as homogeneous as microelectronic circuits.

Independent of their function microelectronic circuits bear a hierarchical composition of standard elements and their inputs, internal processing and outputs are exclusively electrical. This uniform structure is the reason for Moore's law and for the great success of microelectronics technology. Due to the continuous miniaturization of the structures the complexity of microelectronic systems (number of transistors) increases faster than chip area and price. A microsystem however is usually one complex device with electrical and non-electrical inputs and outputs. Its functionality does not scale with the number of elements. And miniaturization of the largest elements like deflecting micromirrors, seismic masses or levers in actuators would reduce performance dramatically.

We will discuss conception, modeling, layout, process design, test and packaging as interrelated phases in the development of microsystems, compare to microelectronics and give examples.

\section{EXAMPLES}

Two of our products will serve as examples to substantiate several statements. This is the micro scanning mirror for laser beam deflection $[2,3]$ and the micromirror array $[4,5]$ for fast optical pattern generation with high resolution (see figure 1).

\subsection{THE MICROSCANNING MIRROR}

The micro scanning mirror is an electrostatic microsystem for the periodic deflection of a laser beam. Its clue is the inplane configuration of driving electrodes, mirror plate and torsion springs in one crystalline silicon layer. A patent [6] was granted for this configuration. The device can scan a laser beam over a range of typically $60^{\circ}$ and higher at driving voltages of typically $20 \mathrm{~V}$ and at very low power consumption. The oscillation is always resonant at frequencies that can be designed to $200 \mathrm{~Hz}$ to $35 \mathrm{kHz}$. Mirror diameter can be between $0.5 \mathrm{~mm}$ and $3 \mathrm{~mm}$.

Fields of application are barcode reading, image capture, laser projection displays [7], endoscopes, pattern generation for triangulation, beam scanning for laser metrology and -if the normally flat mirror is structured as a grating- NIR spectrometry [8]. In particular for the fields of scanned head mounted displays, and endoscopic imaging micro scanners are considered an enabling technology.

\footnotetext{
* The terms MEMS and microsystems are used interchangeably here.
} 
The fabrication process by bulk micromachining in BSOI-wafers (bonded silicon on insulator) is designed for the fabrication of a few 10000 devices per year to several million. First, membranes of crystalline silicon are formed (SOIlayer of typically $30 \mu \mathrm{m}$ ) by local removal of the handle silicon through an anisotropic wet etch in TMAH. Then a DRIE etches deep trenches that define mirror plate, torsion springs and comb electrodes. Before the anisotropic wet etch filled isolation trenches, bondpads, metal interconnections and the aluminum coating for the mirror have been formed on the solid wafers. The fabrication process ends with the functional test on wafer-level. More detailed information is given in $[2,3]$. The micro scanning mirror is a low-cost device with excellent performance. Neither device nor process is particularly complex.

\subsection{THE MICROMIRROR ARRAY (SLM)}

The micromirror array is a pixel matrix of $2048 \times 512$ deflectable $16 \mu \mathrm{m}$-micromirrors on a CMOS backplane. It is a spatial light modulator (SLM) similar to the DLP [9]. But in contrast to the DLP it allows gradual deflection of the micromirrors and operation with DUV light of $248 \mathrm{~nm}$ wavelength. Frame rate is $2 \mathrm{kHz}$ for analog patterns.

The main focus of the development lies on the application in a leading-edge mask writer [5]. The micromirror array is the enabling high-performance devices. Its capability of analog pixel deflection is not used for gray scaling, but for edge shifting in a $2 \mathrm{~nm}$ grid based on a diffractive effect. Only due to this unique feature DUV has become an alternative to the electron beam for leading edge mask writing.

The device is very complex and so is the fabrication process. After the high voltage CMOS process with 4 metal layers, the micromirrors are formed by aluminum surface micromachining with a sacrificial polymer layer. Release and functional test are performed on die-level. The required numbers are not high enough for yield optimization. Also price is of low priority. The objective is device performance. A number of very tight specifications are met.

\section{THE DESIGN PROCESS}

A very good comprehensive textbook on the design of microsystems is [10]. From the point of initiation Senturia distinguishes between market-driven design and technology-driven design. Where technology-driven refers to a technological concept for a microsystem that identifies suitable fields of application, and market-driven means a component in a system is needed that must meet certain requirements, and microsystems are among the options.

A further differentiation is, whether the goal is a commercial product or a technology demonstration. Technology demonstrations usually precede a commercial product no matter if the concept to be demonstrated was made up by itself (technology-driven) or if it was developed from requirements (market-driven). The design of a commercial product is even more complex than demonstrator design, because questions on cost, yields, reproducibility, operating conditions, reliability, capacities and intellectual property must be answered. But also in demonstrator design it is recommendable to answer as many questions for commercial products as possible if there is the vision of a commercial product in the future. Senturia illustrates this complex situation with figure 2 .

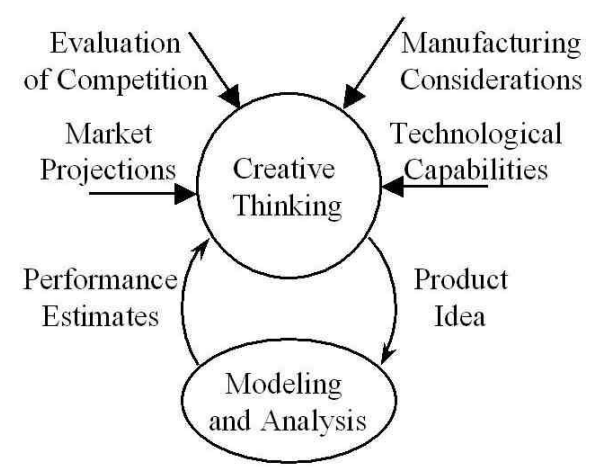

figure 2 The design process for microsystems is still a very complex issue [10, courtesy of Kluwer Academic Publishers] 
The authors suggest starting from the list of requirements and designing the microsystem to meet all specifications. In the technology-driven case it is a good idea, to identify a few representative applications and investigate detailed specifications. Then going the market-driven way and finding solutions that meet the requirements leads to a very helpful attitude.

In the second step we recommend to go again to the application and complete the specifications to a comprehensive list. Operating conditions, environmental conditions during storage, expected product life must be specified. If the design turns out to have only a single important deficit one may be forced to start from the scratch again. Then one should find out about official and nonofficial standards. If the microsystem is supposed to substitute for a conventional component of a system, same dimensions of the package and the same screw in the right position may avoid redesign of the system. This will facilitate evaluation and introduction. And the systems manufacturer reduces his risks, because the way back to the conventional component remains open.

These industry standards exist for several fields like automotive, medical, aerospace or mobile applications. But in addition one should interrogate a system designer from the respective field and discuss his concerns. His questions will have to be answered anyway sooner of later. Addressing many points should reveal also requirements that the insider premises, but forgets to mention.

Every custom-specific redesign of our micro scanning mirror, for example, begins with the analysis of the required frequency, scan angle and mirror diameter. When a plate design with acceptable dynamic deformation is determined, the spring design must secure resistivity against shock and sticking (see below). The required temperature ranges for operation and storage are analyzed later in design phase. This mainly affects packaging. Head-mounted and hand-held devices e.g. can obviously not be operated with high temperatures at the case. Internal temperature however may be a different thing. And if left in a parked car, the device must survive storage temperatures above $70^{\circ} \mathrm{C}$. The micromirror array on the other hand must provide a precise and stable deflection-voltage characteristic at only one temperature. Operation temperature and humidity inside the mask writing machine are carefully controlled. Storage temperature during shipment however must still be considered.

When a detailed concept for the microsystem is found (Product idea in figure 2), the first simulation cycle (modeling and analysis) calculates the behavior of the microsystem (performance estimates) that is compared to the derived list of specifications. After refinement work on concept and models the improvements are calculated. This iterative process goes on until the microsystem, its fabrication and its economics are well understood and a suitable design point is found.

\section{MODELING}

Modeling a system means to find a description of the behavior of the system. The gained knowledge is essential to evaluate a design and to find a suitable design point. Four different levels of abstraction can be identified in the modeling of microsystems as illustrated in figure 3 .

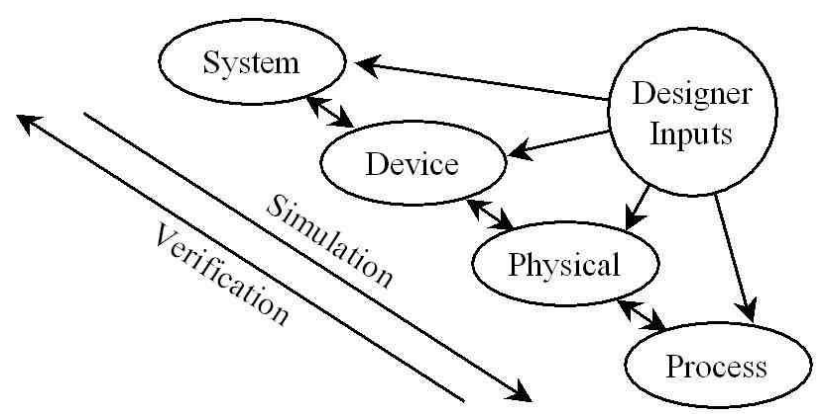

figure 3 Modeling of microsystems [10, courtesy of Kluwer Academic Publishers]

The highest level of abstraction is system level. System modeling helps to derive the requirements of the microsystems device from the requirements of the system. Tolerable deviations of the device behavior can be identified. In the 
example of the micromirror array system modeling investigates the optical projection system, the data path and the signals. Results are specifications for global flatness, position accuracy of mirror elements, tolerable jitter and the minimum number of data ports. For the micro scanning mirror modeling of the optical system derives e.g. maximum tolerable dynamic deformation for a small laser spot.

Device level simulation models the properties of the microsystem device from its structural elements. In the top down approach this may break down the device properties to the properties of the structural elements, e.g. the stiffness of the torsional springs of the micro scanning mirror.

Physical level modeling deals with 3D geometries, material properties and the surrounding medium. This is the domain of FEM simulation. Stiffnesses are calculated for concrete torsional spring geometries of the micro scanning mirror. Dynamic deformation at maximum deflection angle is determined for silicon plates of certain thickness and diameters at the required oscillation frequency. For the micromirror array the deflection voltage characteristic is determined. While the micro scanning mirror lends itself to physical FEM simulation of the entire device, physical simulation of the micromirror array deals with a single mirror element or with a few neighboring pixels in order to model crosstalk.

Process level modeling finally finds the required patterns on the photo masks and how to adjust certain process parameters. So process modeling is investigates the design rules for the layout. For the micro scanning mirror e.g. the required sizing of the torsional springs is estimated (increased spring width on the photo mask to compensate for the under etch in the DRIE). Another example is the size of the backside hardmask for the anisotropic backside wet etch. It leads to the correct dimensions of the membrane where mirror plates, springs and comb electrodes are structured.

Experienced process engineers can actually widely do without any computational simulation. They know the achievable process results better than all the individual parameters of influence. So, discussing a layout with experienced technologists is the best thing one can do. When the geometry is known that will come out of the process, one can draw the layout in a way that the desired geometries are created.

It is a wise idea to calculate the resulting structures for the typical process variations. The geometries then go back into the physical models in order to determine the performance deviations from device to device that must be expected. The results from physical modeling is often parameterized with fit functions or laid down as data tables in order to have a compact description of device behavior suitable for efficient system level simulation.

Much more helpful than numerical simulation are an analytical models. It usually takes some brainwork to derive a set of formula from physical basics that describe a simplified geometry. But the effort pays back when searching for the best design point, because an exact understanding of the dependencies allows straight optimization. For the micro scanning mirror e.g., it is very helpful to know the approximate relation between the resonant frequency $f_{0}$ of the mirror oscillation and the geometrical dimensions of plate and torsional springs [11]:

$$
f_{0} \propto \sqrt{\frac{d^{3}}{\rho \cdot a^{3} \cdot b \cdot l}}
$$

$a$ and $b$ are the mirror plate dimensions. ( $a$ is perpendicular to the torsional axes and $b$ is parallel.) $\rho$ is mass density of the plate material silicon, $d$ is spring width and $l$ is spring length. This relation is valid for straight torsion beams as springs, if spring width $d$ is much lower than the height $t_{\mathrm{SO}}$ of both, the springs and the mirror plate. It is interesting to see that SOI thickness $t_{\mathrm{SOI}}$ has no influence on resonant frequency. Spring width on the other hand is the main influence parameter with $f \propto d^{3 / 2}$. Variations $\Delta d$ of spring width $d$ are an absolute value in the range of $100 \mathrm{~nm}$, while $d$ is in the range of a few microns. A spring width around $d=5 \mu \mathrm{m}$ for example will have variations of $\pm 2 \%$. So frequency variations of $\pm 3 \%$ must be accepted. Wider springs have relatively smaller frequency variations, but double spring width would require 8 times longer springs in order to meet the same frequency.

The discussion of the last paragraph shows that an analytical model gives a clear view on the influence that parameters have on key properties of the device. The best design point can then be discussed in relatively short time. Only for final tuning FEM is employed to consider also the actual spring geometry with design features like tapers. 


\section{LAYOUT}

Layout refers to the geometrical content of the photo masks that is exposed into the photo resist. Critical dimension in microsystems technology is usually not far below $1 \mu \mathrm{m}$. At this scale the transfer of the shapes and dimensions can be considered as 1:1. State of the art CMOS however has critical dimensions below 100nm. At this scale the photo masks contain many features that compensate for optical effects in order create the intended shapes in the resist mask (see figure 4).
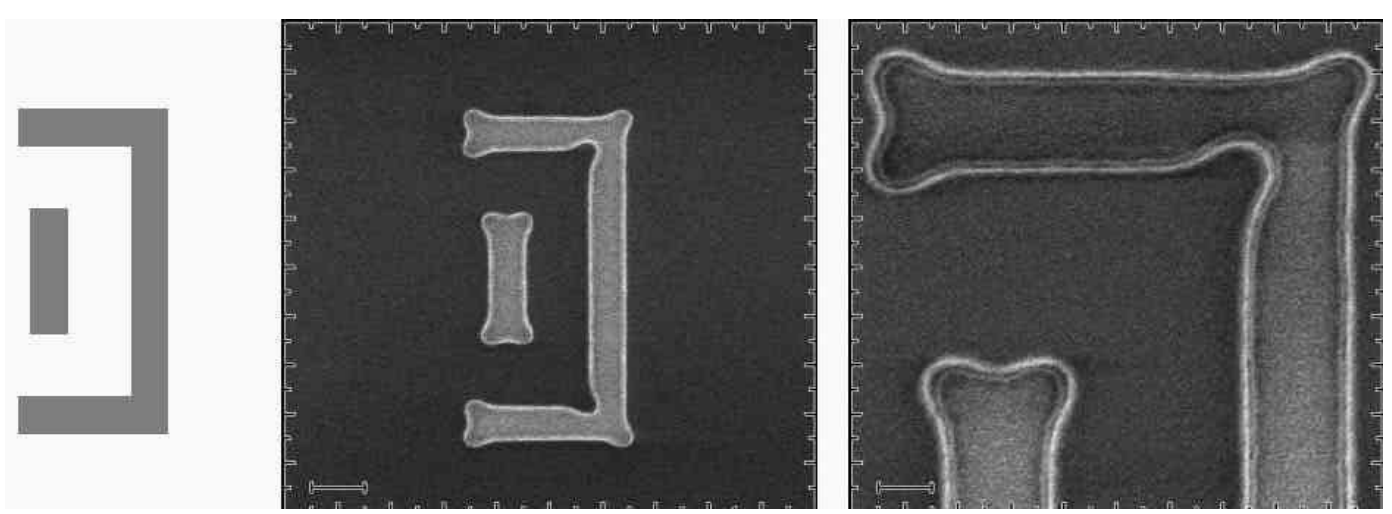

figure 4 Serifs in sub-micron photo masks. For the creation of the structure on the left-hand side. The photo mask must contain the shapes in the middle and on the right-hand side.

The infrastructure of photo mask generation for microelectronics can be used for microsystems without any change. Another re-use of this concept are compensations structure that compensate for shape variations like gradual rounding of corners in long etch processes.

Another instructive concept in CMOS technology is the self-alignment of the gate. In figure 5 the concept is illustrated. Elder processes created the source and drain regions by a diffusion. When the gate metal was deposited and structured exact alignment became crucial with shrinking gate length in order to obtain equal overlaps. The current process accepts poly-silicon as gate material despite of its lower conductivity, because it can be deposited and structured before the implantation of source and drain. In this way the poly-gate acts as a mask that prevents any dopants under the gate and source and drain are exactly aligned to the gate.
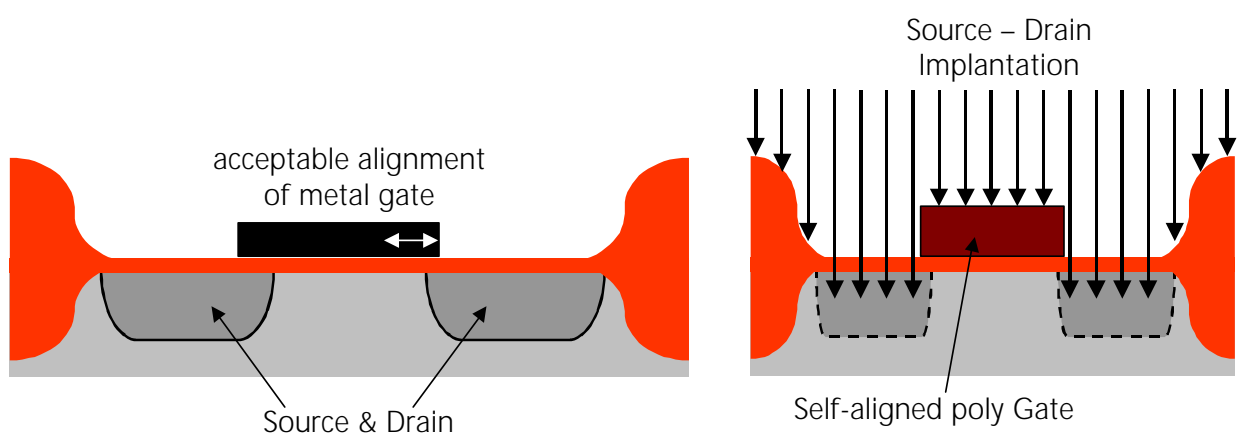

figure 5 Left: At large gate length the alignment of the metal gate to the source and drain diffusions was sufficient. Right: With a polysilicon gate as mask for the source-drain-implantation the gate is perfectly aligned also in short-channel transistors.

Concepts of self-alignment are very powerful also for microsystems, when elements in different technological layer require exact alignment. An example can be found in [12], where electrode combs with an initial vertical displacement are created. This concept is superior to concepts with front-to-backside alignment in a mask-aligner. 
In the micro scanning mirror for example comb-drives, springs and the symmetric mirror plate are all generated in the same DRIE-etch from one photo mask in order to avoid misalignment. This results in excellent symmetry of the oscillating plate and its axes, and in precise alignment of both sides of the interdigitating comb electrodes (figure 6). In addition, also the filled isolation trenches, that provide an asymmetric distribution of the electric potentials, have a symmetrical layout in order to avoid unbalanced dynamic deformations.

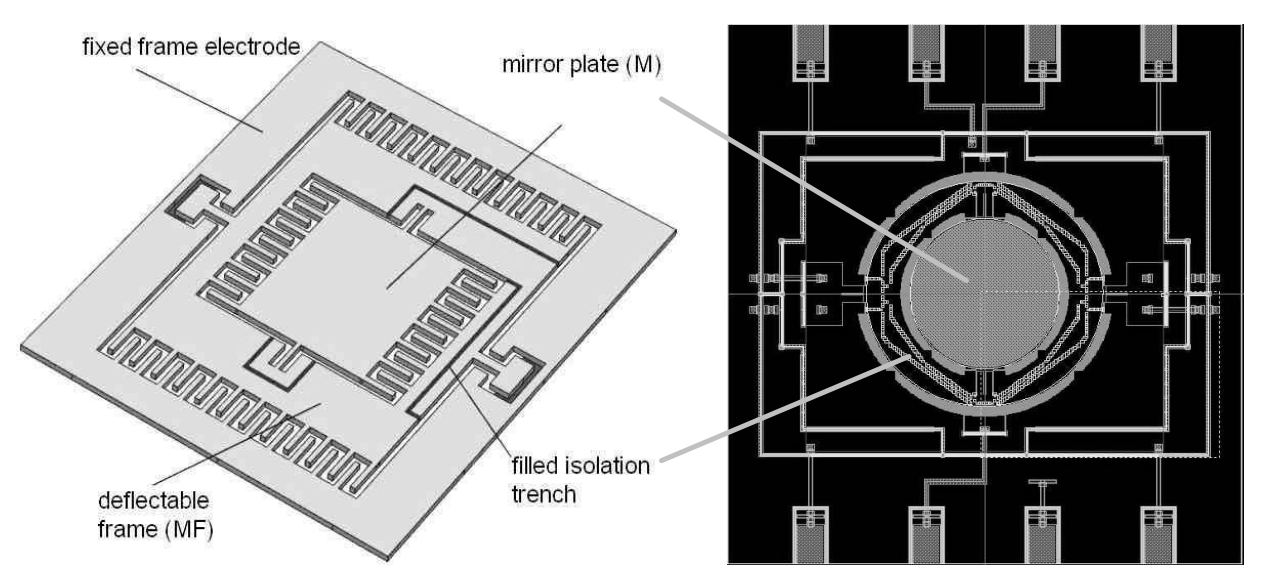

figure 6 Left: The same DRIE etch step defines plate, torsional springs and the comb-drives. Right: In the actual layout of the 2D micro scanning mirror the filled isolation trenches have symmetric paths, although their electrical function is asymmetric (see again left-hand side).

Analog microelectronics often requires pairs of transistors and resistors with exactly the same characteristics. Any difference produces asymmetries e.g. in differential amplifiers. For this reason pairs of elements are placed at minimum distance and with the same orientation as shown in the layout in figure 7. Sometimes the layout of such transistors is even interweaved. The reason is that also local process variations usually have a bigger extension than the transistors. So they affect both elements to exactly the same degree.

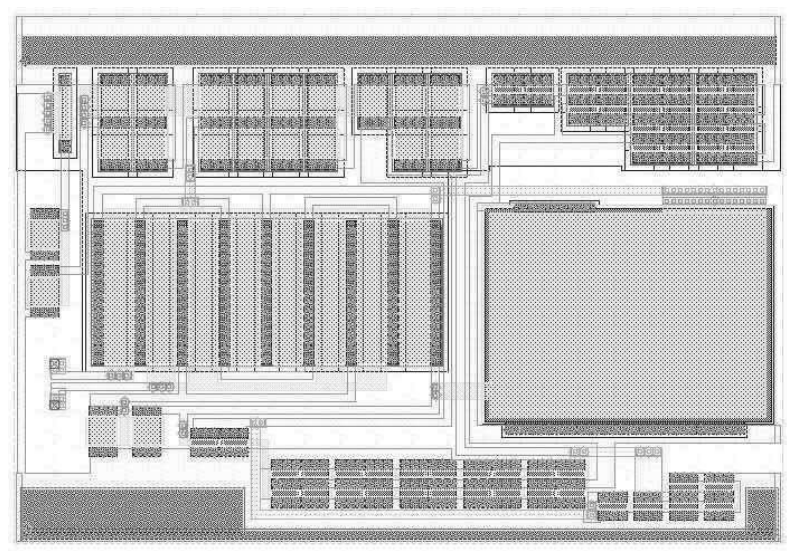

figure 7 Regular structures in the layout of an operational amplifier with transistor pairs placed at minimum distance.

A similar concept in the layout of the micro scanning mirror is the choice of the same spring width for both axes of a 2D-sanner mirror. In this way process variations affect both frequencies in the same way and the frequencies ratio $f_{\mathrm{x}}: f_{\mathrm{y}}$ remains constant.

Further helpful concepts from CMOS technology that should be adopted are test structures that allow evaluation of the success of process steps. Or placing names and identifiers for the mask version into the layout. 


\section{PROCESS DESIGN}

Designers of CMOS circuits just receive a new set of design rules when the technologists have developed the next generation process. In microsystems technology nearly every device has its own fabrication process. So process design (also called process implementation) is still an inherent part of the design of microsystems.

And process design is the most complex part of microsystem design. As explained above the monolithic nature of the fabrication processes has only limited degrees of freedom because of extensive interdependencies. An excellent idea for a new process flow may be spoiled by a single small detail, like a too high process temperature on a wafer with deposited aluminum or like an aggressive cleaning step that destroys features already on the wafer. Then required overlaps and distances (design rules) may lead to uncomfortably large minimum feature sizes. Also features created unintended at the wafer edge are often overseen.

The best start is to understand the principle wafer processes for layer deposition, structuring, etching, oxidation and wafer bonding. Then studying existing process flows and understanding the reasons for the particular order sets up database in the brain for the evaluation of new process flows. And one should not change existing process sequences without good reason.

Next it is vital to have a god overview of the wafer processes that are available in the chosen foundry. A standard process step with only slight variations may just be unobtainable anywhere, if the own foundry does not offer the respective standard step. Also machine details like clamp ring or electrostatic chuck may prevent wafers from being processed. An automatic wafer handler may just not recognize thin wafers, a vacuum handler or support pins may crack membranes as well as the fast evacuation of a process chamber may blow away mechanical elements.

Finally it is complex and creative task to design the process flow, as mentioned under consideration of all the interdependencies and limitations. Discussion with experienced technologists and their perpetual evaluation of the ideas are invaluable.

\subsection{PROCESS VARIATIONS}

Any process is subject to small variations. This means varying dimensions after pattern transfer and varying layer thicknesses after deposition and layer growth. In the particular case of microsystems material parameters of layers play an important role while CMOS circuits are sensitive to varying dopant levels.

Pattern transfer refers to shifting edges of the structures in photolithography and the subsequent etch step. This variation has an absolute nature and is a few $100 \mathrm{~nm}$ for typical equipment in current microsystems technology. Hence large structures $>>10 \mu \mathrm{m}$ vary relatively little, while small structures like narrow torsional springs $<2 \mu \mathrm{m}$ suffer from relatively large variations. So a general design rule for flexure design is: Exploit the space you have! Larger flexures are better reproducible.

An important breakthrough in the resolution of photolithography was the implementation of CMP (chemicalmechanical planarization) in CMOS technology. By eliminating the step heights on the wafer surface all exposed structures where located in the focal plane. Microsystems deal with much higher step heights up to a few microns. If high steps cannot be avoided at all, the designer must ensure that the focal plane is always adjusted properly. Auto focus systems may cause trouble.

For many deposition processes layer thickness has a tolerance of $\pm 5 \%$. This is crucial for cantilevers and membranes in surface micromachining. Typical materials for surface micromachined structures are polysilicon, silicon nitride, aluminum and silicon oxide.

A very good compensation for thickness variations of the crystalline SOI-layer can be found in the design of the micro scanning mirror: torsional springs and mirror plate have the same thickness and the springs are narrow. In this case resonant frequency is almost independent of SOI-thickness.

Many microsystems are particularly sensitive to variations in the material properties of layers. Varying stress and elasticity may cause undefined bending and deflection of cantilevers, plates and membranes. In the example of the 
micro scanning mirror all layers have been abandoned from the $30 \mu \mathrm{m}$ thick mirror plate, except a $100 \mathrm{~nm}$ thin aluminum coating for enhanced reflectance. For this reason the mirror remains flat.

Another approach is the optimization of the deposition process for low stress. This involves also thermal stresscompensation over the temperature range from the operating conditions. However, when dielectric layer deposition is optimized for low stress, the electric strength is certainly not at its optimum any more. This must be considered when the dielectric layers still serve as insulators [13].

In general the design point should tolerate the natural variations of the process. (See also modeling in section 4.) Whenever excessively tight process control is required, cost grows and yield decreases.

\subsection{PROCESS RISKS}

Compared to CMOS wafers, microsystems bear features that are more sensitive to damage. KOH-openings, v-grooves or deep trenches may be kind of a perforation of the wafer, which is more likely to break with mechanical impact. This kind of structures should better be created towards the end of the process. Or be filled until the release process. The same applies to thin wafers. The only reason for having $675 \mu \mathrm{m}$ thick wafers in CMOS is stability in the process. The micro scanning mirror e.g. requires more careful wafer handling than CMOS wafer, while the micromirror array is a standard CMOS wafer with additional surface layers.

Micromechanical elements may be cracked or ripped of in gas or liquid flow or by handling systems. Sacrificial protection layers may provide protection and stiffness. Wafer bonding often prevents mechanical contact. CMOS wafer handling involves significant shocks of a few $1000 \mathrm{G}$ for short duration around $0.1 \mathrm{~ms}$. For this reasons micro scanning mirrors are designed to be shock-resistant until at least $6000 \mathrm{G}$ for $0.2 \mathrm{~ms}$ and $2000 \mathrm{G}$ for $0.7 \mathrm{~ms}$. More fragile designs are not accepted.

Sticking is a frequent problem with microsystems. Small gaps around the microstructures form narrow capillaries where liquids exert strong attracting forces against soft springs. Sticking can be addressed by precautionary surface treatment, by supercritical drying or in the layout by designing only stiff structures that are not bent by the capillary forces. Designs for the micro scanning mirror for example are restricted to a certain lateral stiffness. The micromirror arrays on the other hand have a dry release process in plasma and there is definitely no contact to liquids in the application.

\subsection{PROCESS FOR VOLUME PRODUCTION}

From the technical point of view a fabrication process is suitable for volume production, if quality of the product is reproducible and yield is stable. This is commonly validated by a qualification in the sense of ISO 9001, that reviews the fabrication line, process documentation, test methods and the reproducible quality over several fabrication lots.

A production line for wafer processing must provide machines in defined conditions (parameter settings and chamber conditioning), continuous control the processes (frequent verification of reproducible process results), logging of all maintenance work and every executed process and clear responsibilities. One can do R\&D in a production line by accepting a lot of restrictions and bureaucracy. But fabrication in an R\&D line is only possible if it is run like a production line. Any influence between different processes or from experiments must be prevented. Of course, the situation is most comfortable if one process fills and pays a line all its own. This is actually the reason why CMOS fabrication achieves very high yields: Usually only one process is run for a variety of products. And even "different processes" do not differ a lot. Microsystem technology, however, must deal with a new process for almost every new product.

Process documentation must contain instructions for all operations, all parameter sets and instructions and criteria for all inspections. If a process step is difficult do describe, this is a clear hint that it is not well understood and needs analysis.

Frequent inspections reveal errors as early as possible. Repair may be possible by means of a rework. Or if the wafers have to be discarded, substitute wafers can be started and the machines can be re-adjusted before the next lot is processed. All data must be recorded and at least information that is correlated to the quality of the end product demands steady statistical analysis for drift and instabilities. Diligent development of test structures is a good investment. Test structures help to evaluate process steps objectively and quantify the results. Thus target values and fail/pass criteria can be defined. 
As stated in section 6.1 the product specification should be met regardless of all process variations within the machines' tolerances. Modeling the influence of the actual process variation on the characteristics of the product is a good evaluation of the potential of a fabrication process. And it helps to identify the process steps that deserve further development. If the fabrication process of the microsystem cannot achieve the required precision, means of calibration must be foreseen in the system.

Tolerances usually describe process variations with statistical distribution. So even low yields may be stable, if the majority of the devices works, but only a few meet some tight specifications. Process risks like the examples discussed in section 6.2 may occur and disappear incidentally. Any such failure deserves analysis, even if it has appeared only once. As long as such an appear-and-disappear failure is not fully understood, the risk is, that it appears again for a longer period. As soon as the failure is understood in a way that it can be activated and deactivated "on demand", it can be permanently avoided.

To recapitulate: A well-designed process can accept all machine tolerances and does not comprise any undetermined risks.

After qualification the process must remain stable. This requires a certain throughput. (We consider 144 wafers per year a reasonable minimum.) This means when calculating fabrication cost and prices, a quite high minimum numbers of devices are to be requested and paid. So a process capable of fabricating different products is definitely an advantage on the market.

\section{TEST}

Test cost is often in the same order of magnitude as fabrication cost for a microsystem. In CMOS technology efficient testing on wafer-level is standard. Adoption for microsystems is challenging, because of their non-electrical inputs and outputs. Further they are still sensitive to particle in contrast to CMOS circuits with passivation. In addition to the efficiency of wafer-level testing cost for the packaging of bad devices can be save. So the development of a wafer-level test is highly recommendable for every microsystem.

The design of the micro scanning mirror has the advantage that the functional test can be fully performed on wafer-level without any wafer bonding or packaging. This was a big advantage already in development, because statistical data gave valuable information on the process.

The CMOS backplane of the micromirror array passes the standard CMOS end-test. The extensive functional end-test however must be performed on die-level after the release process. For the relatively low numbers this is acceptable. An example for a design for testability in the layout of the micromirror array are extra pixels outside the matrix with extra test pads. They allows precise measurement of pixel characteristics.

\section{PACKAGING}

Microelectronics technology has developed standard packaging solutions for various requirements, like low cost, extended temperature range or low electromagnetic immission. The performance increase of the last decade however has stimulated a diversification with for high power-dissipation, automatic PCB assembly, compact dimensions, chipon-board, wafer-level packaging, multi-chip modules and other developments.

Microsystems technology has even more diverse requirements. The packaged microsystem must have access to the electrical as well as to the non-electrical signals. Optical window or fiber connectors are required for optical MEMS, pressure sensors need openings to the environment, the micro scanning mirror requires a large cavity for free motion. And in addition microsystems require much more delicate handling, than microelectronic circuits. In particular the dicing process is critical with microsystems. The dilemma is usually that the mechanical elements must be protected from debris and released on die-level. For this reason wafer-level packaging is very attractive. On the other hand this usually implies a separate development. Also the few companies that offer this service for microsystems do not have versatile standard solutions. E.g. [15]. 
It is difficult to give general guidelines. But due to the lack of standard solutions a separate packaging solution must be planned. The development will start with the analysis of the requirements. And it should start together with the development of the microsystem and be interlinked with it, because the dependence between the design of the microsystem and the package is strong and mutual.

Although our examples are both optical microsystems the packaging requirements are quite different. Micro scanning mirror as a compact low-cost device requires an inexpensive and compact packaging solution suitable for a high degree of automation. The micromirror array on the other hand requires a sophisticated ceramic package with high mechanical precision, very low temperature drift and a high number of interconnections.

\section{SUMMARY}

We have discussed the development of microsystems with focus on a design for volume manufacture. Microsystems design was found to be particularly complex, because of the monolithic fabrication process. And in addition the design of a new microsystems nearly always involves process development and packaging development.

The first step is finding and analyzing all requirements. Extensive modeling of system, MEMS component and fabrication process then evaluates the concept. Modeling process variations, gives an early idea of achievable tolerances and yields. Layout generation for microsystems follows the same principles as microelectronic layout. Only sophisticated software for automation is not yet available.

Process design is the most complex part of microsystems design, because of numerous interdependencies between the process steps. The availability of all process steps must be considered as well as natural process variations and inherent risks. A manufacturing process is commonly validated by a qualification, that reviews the fabrication line, process documentation, test methods and the reproducible quality over several fabrication lots.

Test and packaging of microsystems tries to adopt solutions from microelectronics. Significant extensions are required, however. The development of a test on wafer-level is highly recommended. Microsystems packaging has no standard solutions. It must be developed together with every microsystem starting at an early stage of development.

The discussed issues are compared to CMOS technology. And two optical microsystems serve as examples: The micro scanning mirror for laser beam deflection and the micromirror array for fast optical pattern generation with high resolution.

\section{REFERENCES}

1. J.-C. Eloy: “Overview of MEMS and MOEMS industry”,Proc. SPIE 5348, Jan 2004, San Jose, CA

2. H. Schenk, et al.: "A new driving principle for micromechanical torsional actuators", Micro Electro Mechanical Systems 1999, New York, NY : The American Society of Mechanical Engineers ASME, 1999, S.333-338

3. A. Wolter et al., "The MEMS micro scanning mirror for barcode reading: From development to production", Proc. SPIE 5343, Jan 2004, p. 32-39

4. H. Kück et al.: „Deformable Micromirror Devices as Phase Modulating High Resolution Light Valves”, Sensors and Actuators A54, 1996, 536-541.

5. U. Dauderstädt et al.: „Application of Spatial Light Modulators for Microlithography”, Proc. SPIE 5348, Jan 2004, p. 119.126

6. European Patent EP 1123526 B1

7. K.-U. Roscher et al.: "Low cost projection device with a 2-dimensional resonant micro scanning mirror", Proc. SPIE 5348, Jan 2004, p. 22-31

8. H. Grüger et al.: "Realization of a spectrometer with micromachined scanning grating", Photonics Fabrication Europe, Brügge, Belgium, SPIE vol. 4945A-6, Nov 2002

9. L.J. Hornbeck et al.: "Digital micromirror device - commercialization of amassively parallel MEMS technology", Proc. of the ASME congress 1997, Dallas, DSC-Vol. 62/HTD-Vol. 354, Micromechanical systems (MEMS), p. 3-8

10. S. Senturia: "Microsystem design", Kluwer Academic Publishers, 2001, 689 pages (in particular, chapters 1, 2, 4, 17; figures 2.1, 2.2, 17.1) 
11. H. Schenk, et al.: "Design and modeling of large deflection micromechanical 1D and 2D scanning mirrors", SPIE Proc. 4178, 18-19 Sept. 2000, Santa Clara, Calif., pp. 116-125

12. D.T. McCormick et al.: "Multiple layer asymmetric vertical comb-drive actuated scanning mirrors", IEEE-LEOS Internat. Conference on Optical MEMS, Waikoloa, Hawaii, USA, Aug 2003, conf. proc, p. 12-13

13. H. Shea et al.: "Design for reliability of drift-free MEMS micromirrors", Photonics Europe 2004, Strasbourg, France, SPIE vol. 5455, Apr. 2004

14. A. Wolter et al.: "Testing of the micro scanning mirror", Photonics Europe 2004, Strasbourg, France, SPIE vol. 5455, Apr. 2004

15. D. Weiss et al.: "An Integrated Cavity Wafer Level Chip Size Package for MEMS Applications, Conf.: Micromachining and Microfabrication, San Francisco, Proc. of SPIE Vol. 4557, p. 183 -191 (2001) 